\title{
Correction to: Assessing Tissue-Specific Autophagy Flux in Adult Caenorhabditis elegans
}

\section{Jessica T. Chang, Malene Hansen, and Caroline Kumsta}

\section{Correction to:}

Chapter 17 in: Sean P. Curran (ed.), Aging: Methods and Protocols, Methods in Molecular Biology, vol. 2144, https://doi.org/10.1007/978-1-0716-0592-9_17

Owing to an oversight, the word "Assessing" in the title of Chapter 17 was spelt incorrectly in the book. The same has been corrected throughout the book. 